Note: This is a preprint of a paper being submitted for publication. Contents of this paper should nut be quoted nor referred to without permission of the author(s).

CONF $-901105--60$

DE91 006332

[To be published in the Surface Chemistry and Beam-Solid Interactions, Proceedings of the 1990

Fall Meeting of the Materials Research Society, Boston, Massachusetts,

November 26-29, 1990]

\title{
IN SITU LASER ABLATION PLASMA DIAGNOSTICS IN THE FILM GROWTH REGIME - EFFECTS OF AMBIENT BACKGROUND GASES
}

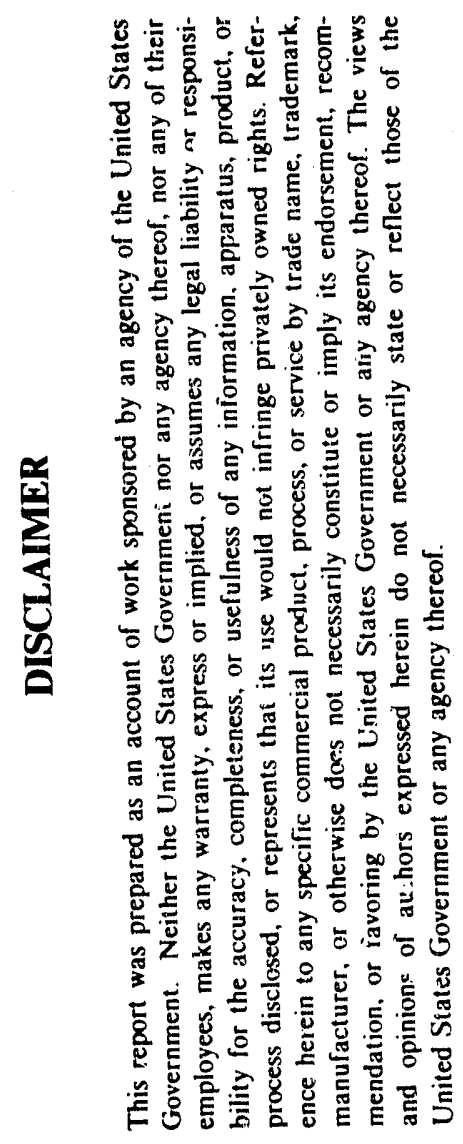

\author{
D.B. GEOHEGAN \\ November 1990 \\ The submitted manuscript has been \\ authored by a contractor of the U.S. \\ Government under contract No. DE \\ AC05-84OR21400. Accordingly, the U.S \\ Covernment retains a nonexclusive, \\ royalty-free license to publish or reproduce \\ the published form of this contribution, or \\ allow others to do so, for U.S. \\ Government purposes."
}

Solid State Division

Oak Ridge National Laboratory

Oak Ridge, Tennessee 37831-6056 operated by

MARTIN MARIETTA ENERGY SYSTEMS, INC. for the U.S. DEPARTMENT OF ENERGY under contract DE-AC05-84OR21400 


\title{
IN SITU LASER ABLATION PLASMA DIAGNOSTICS IN THE FILM GROWTH REGIME - EFFECTS OF AMBIENT BACKGROUND GASES
}

\author{
D.B. GEOHEGAN \\ Oak Ridge National Laboratory, Solid State Division, Oak Ridge, TN.
}

\begin{abstract}
The propagation of the laser-induced plasma formed by $\mathrm{KrF}$ irradiation of $\mathrm{Y}_{1} \mathrm{Ba}_{2} \mathrm{Cu}_{3} \mathrm{O}_{7}$ has been characterized in background pressures of oxygen and argon typically used for thin film growth. The ion current transmitted through the background gases was recorded along the normal to the irradiated pellet as a function of distance in order to measure the decreasing velocity and magnitude of the expanding plasma current due to collisional slowing and attenuation of the laser plume. The integrated ion charge delivered to a substrate at low pressures can be described by elastic scattering giving a general integral cross sections of $\sigma_{\mathrm{e}}\left[\mathrm{O}_{2}\right]=3.2 \times 10^{-16} \mathrm{~cm}^{2}$ and $\sigma_{\mathrm{e}}[\mathrm{Ar}]=2.7 \times 10^{-16} \mathrm{~cm}^{2}$. Ai higher pressures, inelastic scattering leads to increased recombination and reactive conversion of ions indicated by increased fluorescence of all the species, which becomes dominated by fluorescence of $Y O$ and $\mathrm{BaO}$. Spatially resolved fluorescence measurements indicate that the luminous boundary to the plasma follows a weak shock front which coincides with the ion flux propagation. The ion transmission is found to drop exponentially with distance and background pressure, in agreement with a simple scattering model which yields general scattering cross sections for ionargon $\sigma_{\mathrm{i}-\mathrm{Ar}}=2.1 \times 10^{-16} \mathrm{~cm}^{2}$ and ion-oxygen $\sigma_{\mathrm{i}-\mathrm{O}_{2}}=2.3 \times 10^{-16} \mathrm{~cm}^{2}$ interactions in background pressures up to $300 \mathrm{mTorr}$. The general features of the plume deceleration are described in terms of a drag force model.
\end{abstract}

\section{INTRODUCTION}

The expansion of the laser produced plasma from a multicomponent target in vacuum can be regarded as an assemblage of supersonic free expansion beams, with individual stream velocity and kinetic energy distributions determined from the surface layer temperature. However, experimental measurements reveal hyperthermal beams of atoms and ions with similar center-of-mass velocities as well as fluorescence far downstream which indicates the persistence of collisions even in the "collisionless" regime. Collisions in a dense Knudsen layer in the early stages of expansion were used by Kelly and Dreyfus [1] to explain the observed common centerof-mass velocity.

Fluorescence from excited neutrals and ions, the most easily observed diagnostic of plume transport, results principally from plasma kinetics due to the ions and electrons in the plume, and does not represent the full picture of plume transport such as the non-emitting ground state species [2]. Ion probes and absorption spectroscopy allow one to observe components of the non-emitting species in the plume: ions which have not recombined or converted and neutrals which have relaxed to their ground states [3].

In situ processing of $1: 2: 3$ superconductor films requires relatively low pressures (typically 10-300 mTorr) of background oxygen in the chamber during deposition. Under these conditions, the plume fluorescence increases, oxides are preferentially formed and the deposition rate falls due to collisions between the plume and the background gas [4-6]. Here, collisional effects dominate as atoms and ions with most-probable energies of $\sim 40-70 \mathrm{eV}$ are reduced to energies of $<1 \mathrm{eV}$ at film growth distances [7]. The situation for accurately modeling the process is extremely complicated. Individual cross sections for the various collision processes are highly velocity dependent and generally unknown.

In this paper, the general features of ion transmission from $\mathrm{KrF}$-irradiated $\mathrm{Y}_{1} \mathrm{Ba}_{2} \mathrm{Cu}_{3} \mathrm{O}_{7}$ through background gases of oxygen and argon are measured by time-of-flight analysis of ion probe waveforms. The magnitude and spatial position of the plasma front are deicninined versus distance and pressure over the range of interest for thin film growth and general scattering cross sections are determined. The collisional slowing of the plume is compared to both blast-wave theory and a drag-force model. 
The experimental apparatus has been described before [6] and consists of a stainless steel. turbopumped bell jar which was typically evacuated to $2 \times 10^{-6}$ Torr prior to backfilling with argon or oxygen. A Lambda Physik EMG 210 excimer laser provided 50ns FWHM pulses of $248 \mathrm{~nm}$ radiation which were apertured and focused by a single cylindrical lens (focal length 50 $\mathrm{cm}$ ) at a $60^{\circ}$ incident angle onto a rotating, high density $\mathrm{Y}_{1} \mathrm{Ba}_{2} \mathrm{Cu}_{3} \mathrm{O}_{7-x}$ target. The beam was focused to a line of $1 . C \mathrm{~cm}$ width on the target, with a burn area of $0.04 \mathrm{~cm}^{2}$. Typically, $100 \mathrm{~mJ}$ was transmitted onto the pellet, producing energy densities of $2.5 \mathrm{~J} \mathrm{~cm}^{-2}$ and peak intensities of $50 \mathrm{MW} \mathrm{cm}-2$. The beam energy was monitored on a shot to shot basis with a beamsplitter and pyroelectric detector (Gentec ED-200). The line focus geometry produced a plume which expanded little along the long dimension of the line, providing a nearly constant $1 \mathrm{~cm}$ absorption length for the optical absorption measurements.

The visible emission from the plume was spatially and spectrally resolved by imaging the plume onto the entrance slits of a 1-meter spectrometer with photomultiplier. The time response of the emission, CW lamp absorption or ion probe current was captured by a transient digitizer (Tektronix 7912AD). Typically ten waveforms were ayeraged for a given collection condition. The time responses of the photomultiplier and the ion probe circuit were 0.3 and $3 \mathrm{~ns}, \ldots$ respectively.

Two types of ion probes were employed for measurements of the positive ion flux along the target normal. A copper planar probe housed $2 \mathrm{~mm}$ behind a grounded $\mathrm{Cu}$ plane with $0.02 \mathrm{~cm}^{2}$ aperture was employed with different meshes attached. Alternatively, a cylindrical copper Langmuir probe of the same area provided identical results. Under most of the conditions described here, the plasma density was sufficiently high to prevent separating the plasma with ever the finest meshes. The ion probe was floated with respect to the chamber and pellet by terminating the coaxial signal line at the digitizer and by providing the bias with a completely isolated and shielded $1 \mathrm{mfd}$-capacitor-bypassed $300 \mathrm{~V}$ battery and potentiometer in series with the $50 \mathrm{ohm}$ signal line.

\section{RESULTS AND DISCUSSION}

Series of ion probe current-voltage characteristic curves were examined in order to insure operation of the probes in the ion saturation (electron repelling) regime. In the same manner as defined for a dc plasma, a characteristic current-voltage curve for the flowing, pulsed plasma can be defined by measuring the current at a given time (arbitrarily the time of peak ion current). Alternatively, the integrated current in the pulse can be measured, less partial to changes in the time dependence of the signal. It was found that a bias of a few negative volts repelled most of the electrons from reaching the probe, and that complete saturation of the ion current resulted from biases greater than $-70 \mathrm{~V}$. However, as the bias was made positive, the electron current increased linearly, not saturating up through the highest voltages measured of $+400 \mathrm{~V}$. This behavior contradicts the typical dc plasma current-voltage curves where a breakpoint is established when the probe repels all ions and acquires a negative space charge sufficient to limit the electron flow. Apparently the nature of the flowing plasma tends to limit the buildup of this negative space charge and no electron saturation is observed. The ion probes for the following measurements were operated in the ion saturation regime with biases of typically - $100 \mathrm{~V}$ in order to measure the absolute number of ions collected over the $0.02 \mathrm{~cm}^{2}$ probe area.

At low oxygen background pressures ( $0.01-10 \mathrm{mTorr})$, the transmitted ion pulse undergoes predominantly elastic scattering. Ions are removed from the beam without significant modification of the velocity distribution. Figure 2a shows the effect on the ion probe signal at $\mathrm{d}=10 \mathrm{~cm}$ in oxygen. The total number of ions in the pulse exponentially decreases with pressure. In addition, the velocity distribution does not vary significantly over the $0-10 \mathrm{~m}$ Torr region as indicated by the normalized 0.01 and 10 mTorr waveforms of Fig. $2 b$.

This situation can be modeled by the classical theory of elastic scattering, where a general integral cross-section is defined for the scattering process by $I=I_{0} \exp \left(-n_{B} \sigma t\right)$, where $I$ and $I_{0}$ are the beam intensities, $n_{B}$ the background number density, $L$ the interaction distance and $\sigma$ is the cross section which describes the scattering. In reality the cross sections are velocitydependent, but can be assumed constant in the approximation that the velocity does not change.

Integral cross sections for oxygen and argon for the situations of Fig. 1 are very similar, $3.2 \times 10^{-16} \mathrm{~cm}^{2}$ for oxygen and $2.7 \times 10^{-16} \mathrm{~cm}^{2}$ for argon. The cross section describing the 

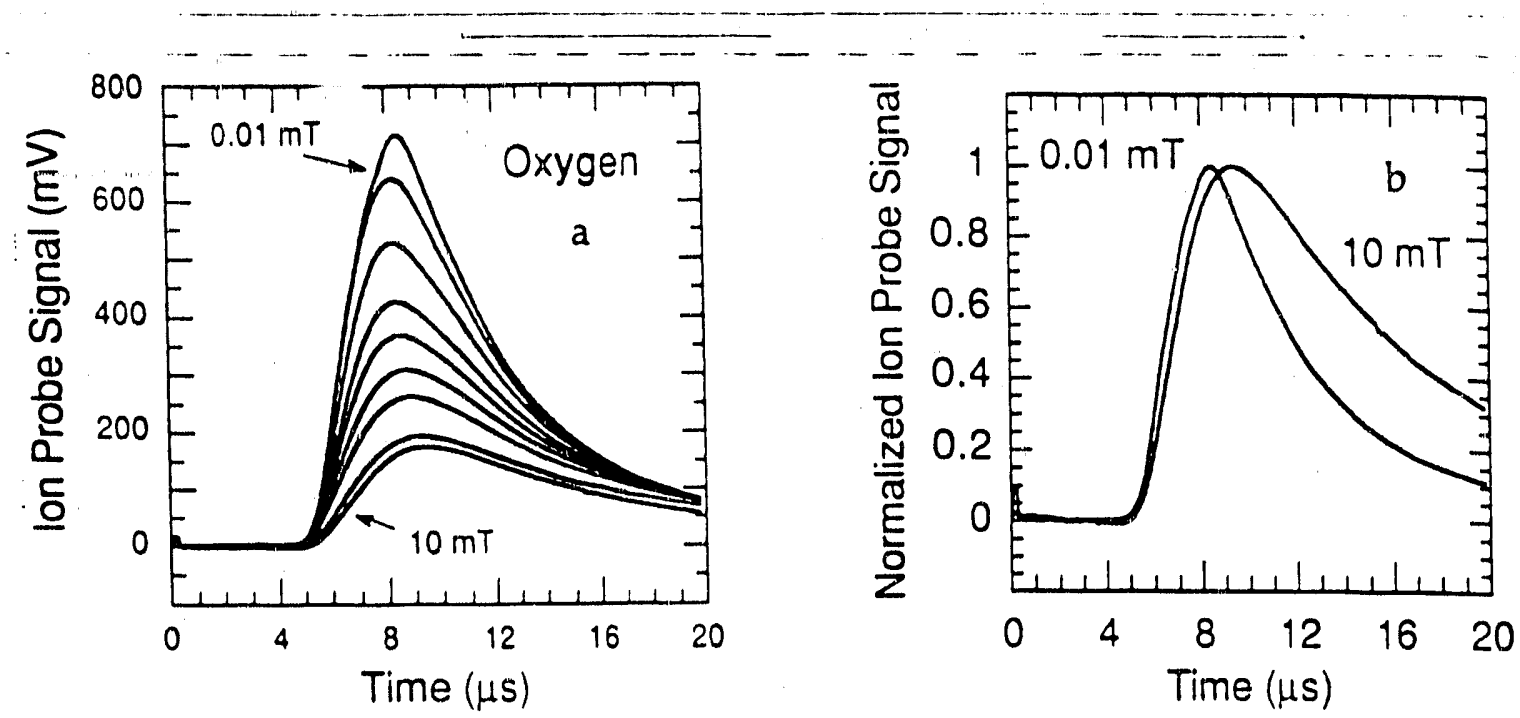

Figure 1.- (a) Positive ion current collected $10 \mathrm{~cm}^{-}$from a $\mathrm{Y}_{1} \mathrm{Ba}_{2} \mathrm{Cu}_{3} \mathrm{O}_{7}$ targer Irradiated ${ }^{-}$with $3 \mathrm{~J}^{-\mathrm{cm}^{-2}}$ at $\mathrm{t}=0$ in $0.01,1,2,3,4,5,6,8$, and $10 \mathrm{mT} \mathrm{O}_{2}$. The integrated charge and peak current decrease exponentially with pressure. (b) N.rmalized current pulses for 0.01 and $10 \mathrm{mT} \mathrm{O}_{2}$ showing the variation in velocity due to collisions.

attenuation of the ions in the ablation pulse includes not only the elastic scattering of ions out of the beam, but presumably conversion of ions into excited neutrals and oxides, evidenced by the increase in fluorescence in the chamber.

At higher pressures, significant slowing of the beam occurs with increased pressure, as shown in Fig. 2. The magnitude of the transmitted ions drops exponentially with pressure as before, but the velocity of the plasma front becomes significantly reduced as well. In addition, two components to the ion flux are observed over a limited pressure range, indicating different slowing rates-for different-ion species.

When argon replaced oxygen at identical pressures, the arrival time and shape of the ion probe signal remained nearly the same, however the magnitude was typically 2 to 4 times greater. Since the two background gases were of nearly the same atomic weight, the slowing of the gas appears to depend directly on the mass of the gas while the magnitude depends additionally on the reactivity.

The transmission of ions at backpressures of 50-300 mTorr was measured for both argon and oxygen at distances from 2.0 to $10.0 \mathrm{~cm}$. The maximum range of the ions was limited by the exponential drop in transmitted intensity with distance and the observed slowing. Ground state Cu neutrals, observed by optical absorption, were also slowed similarly. In general, the transmission of the plume as a whole was well represented by the ion probe transmission.
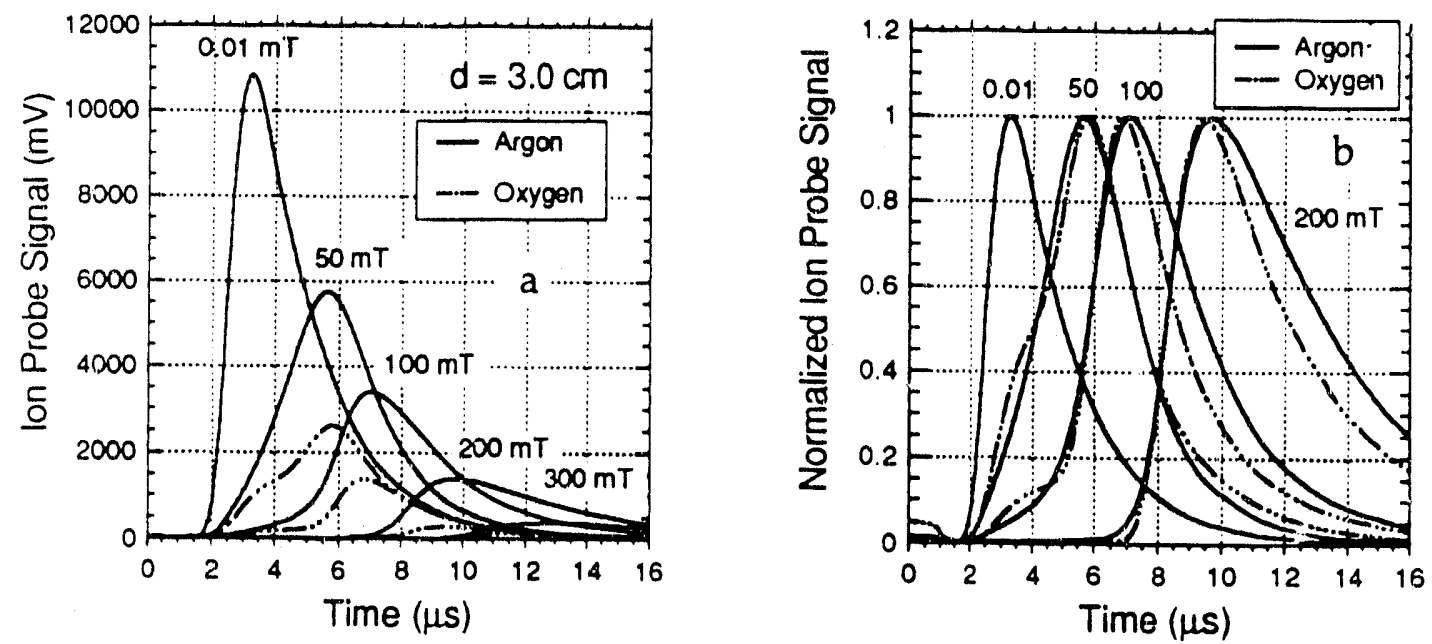

Figure 2. (a) Comparison of ion current waveforms $3.0 \mathrm{~cm}$ from $\mathrm{KrF}$ irradiated $\mathrm{Y}_{1} \mathrm{Ba}_{2} \mathrm{Cu}_{3} \mathrm{O}_{7}$ in 0.01 and 50.100.200. and $300 \mathrm{mT}$ background pressures of argon and oxygen. (b) Normalized $0.01,50,100$, and $200 \mathrm{mT}$ waveforms showing similarity in collisional slowing effects for oxygen and argon. 

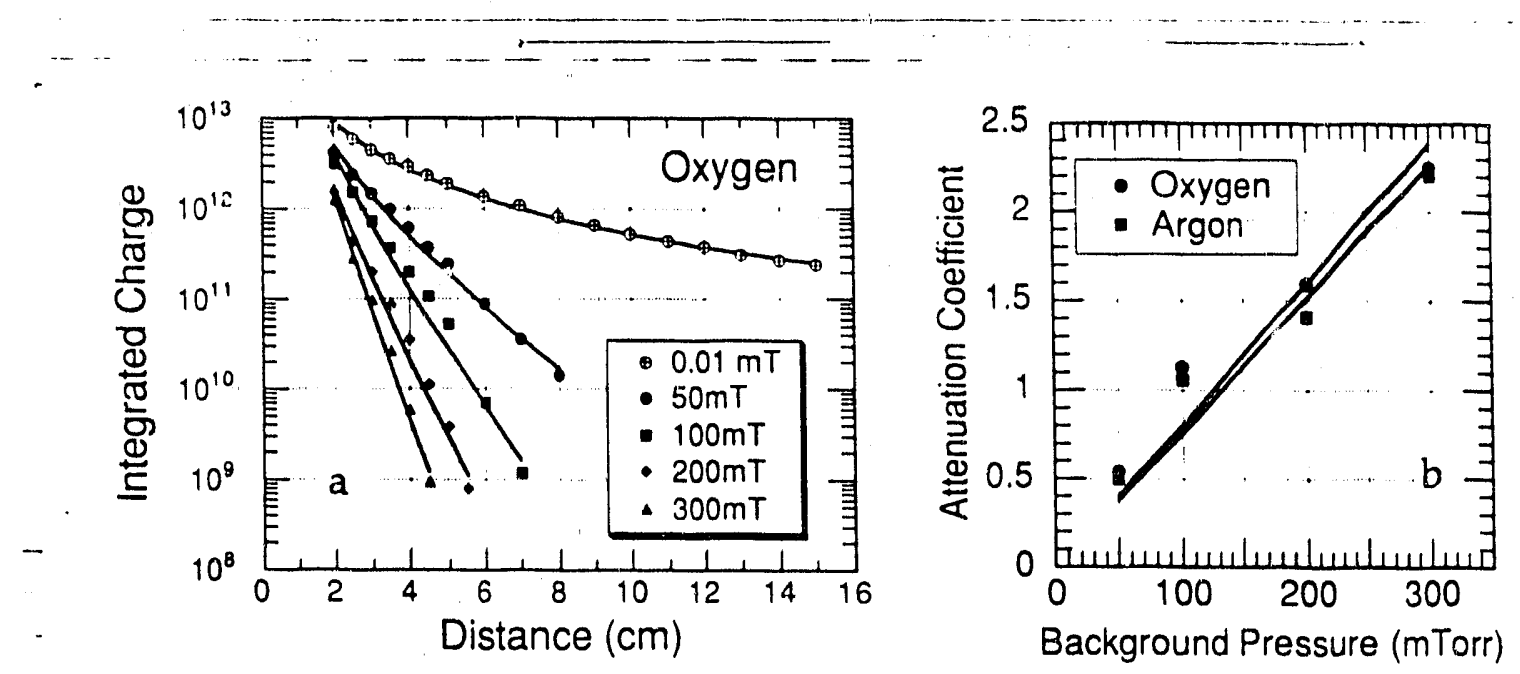

Figure 3. (a) Transmitted-total-ion charge-through oxygen-backpressures of $-0.01,-50,-100,200 \ldots$

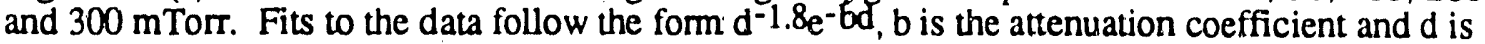
the distance from the pellet. (b) Plot of the experimentally determined attenuation coefficients versus background pressure yielding scattering cross sections of $2.3 \times 10^{-16} \mathrm{~cm}^{2}$ (oxygen) and $2.1 \times 10^{-16} \mathrm{~cm}^{2}$ (argon).

The integrated ion charge delivered to the ion probe for various distances are shown in Fig. 3a. In vacuum the ion flux drops as $\mathrm{d}^{-1.8}$, in near agreement with the expected $\mathrm{d}^{-2}$ drop for a detector of constant size at distance $d$ from the origin of the nearly two-dimensional expansion. Using the vacuum density for $\mathrm{I}_{0}$, the attenuation of the integrated charge were fit to $I=a I_{0} \exp (-b d)$, where $b$ represents the attenuation coefficient. The attenuation coefficient was found to depend linearly on background pressure as shown in Fig. 4b where the slope of the curve yields the generat scattering cross section for ion-argon $\sigma_{i-A r}=2.1 \times 10^{-16} \mathrm{~cm}^{2}$-andionoxygen $\sigma_{\mathrm{i}-\mathrm{O}_{2}}=2.3 \times 10^{-16} \mathrm{~cm}^{2}$ interactions for background pressures up to $300 \mathrm{mT}$ Torr. Although not shown explicitly here, this attenuation appears to be mimiced by the amount of collected film deposited at a given distance, yielding a good measurement of the relative deposition rate under different background pressure conditions.

As shown in Fig. 3b, while the overall ion attenuation rates are nearly equal in oxygen and argon for distances $>2 \mathrm{~cm}$, the magnitude of the number of ions reaching $\mathrm{d}=2 \mathrm{~cm}$ is significantly reduced in the reactive oxygen environment (see Fig. 2a). The ratio of preexponential coefficients [a $\mathrm{Ar} / \mathrm{aO}_{2}$ ] from $\mathrm{I}=\mathrm{a} \mathrm{I}_{0} \exp (-\mathrm{bd})$ increases from 1.6 at $50 \mathrm{mTorr}, 2.0$ at $100 \mathrm{mTorr}, 2.3$ at $200 \mathrm{~m}$ Torr, to 4.02 at $300 \mathrm{mTorr}$. While the emission from neutrals, ions and oxides increased due to increased collisions in either background gas, gated emission spectra showed greater increase in oxide fluorescence vs. atomic lines and approximately twice as much integrated oxide fluorescence from $50-400 \mathrm{~m}$ Torr with oxygen instead of argon.

This reduction in ion intensity and increase in oxide fluorescence in oxygen is illustrated in Fig. 4 where the temporal ion flux and $5972 \AA$ YO fluorescence intensity at $\mathrm{d}=2.0 \mathrm{~cm}$ are plotted vs. time. At $0.01 \mathrm{mTorr}$ in vacuum the ion density is unattenuated and there is negligible oxide fluorescence. At $50 \mathrm{mTorr}$, collisions begin to attenuate the ions and $\mathrm{YO}$ is produced during the slower component of the ion pulse. At higher pressures, the ions continue to decrease and the oxide fluorescence peaks at 200 mTorr and then falls. At pressures $>150 \mathrm{mTorr}$, the leading edges of the plasma front and the $\mathrm{YO}^{*}$ fluorescence were found to coincide.

The ion probe signal monotonically decreases with increasing pressure and distance, while the inelastic processes result in conversion of the ions into excited states and oxides, peaking their densities at a given distance and pressure. The leading edge fluorescence of all observed species were found to follow the leading edge of the ion pulse at pressures $>200 \mathrm{mTorr}$. Above $200 \mathrm{mTor}$ and distances of $2 \mathrm{~cm}$; the ions; neutrals and oxides appear to converge into a weak shock structure with common velocity, collisionally equilibrated and coincident with the ion probe signal.

The progression and slowing of the plasma front in backgound pressures of oxygen and argon was measured by recording the arrival time of the ion probe signal peak at various. disiances. The resuitis are given in Fig. 5. The siope of a smooth curve drawn through the points gives the instantaneous velocity of the plasma peak at a given time and distance 

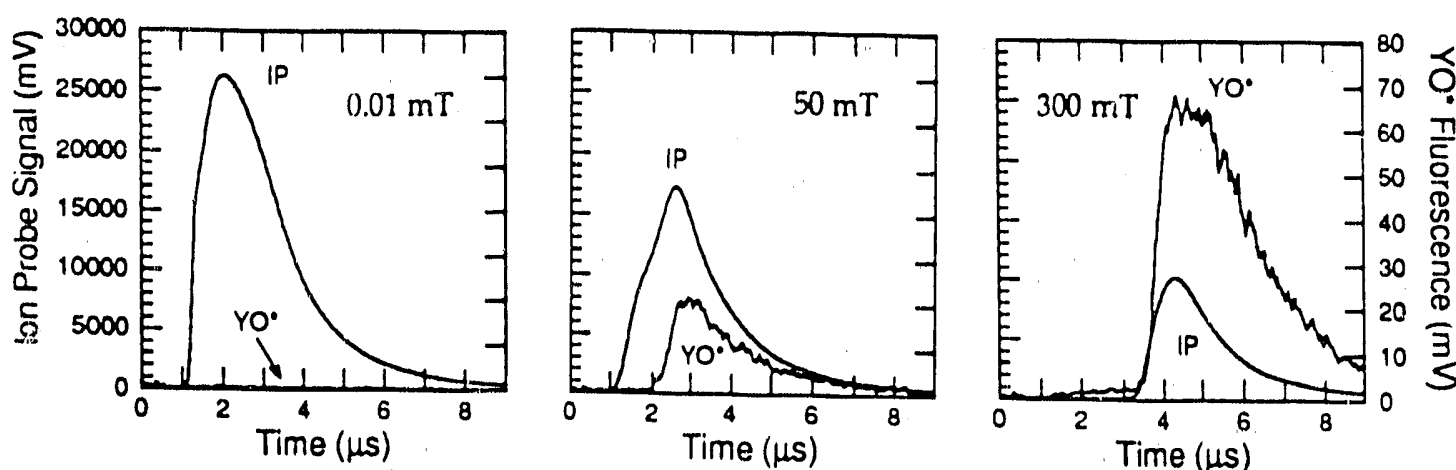

Figure 4. Ion flux and $\mathrm{YO}^{*}$ fluorescence $(5972 \AA)$ at $\mathrm{d}=2.0 \mathrm{~cm},\left(\mathrm{KrF}, 2.5 \mathrm{~J} \mathrm{~cm}^{-2}, \mathrm{YBCO}\right)$ in $0.01,50$ and $300 \mathrm{mT}$ oxygen. The slowing and attenuation of the ion pulse is accompanied by an increase in oxide emission and a coincidence of the leading edges of the pulses for P> $150 \mathrm{mT}$.
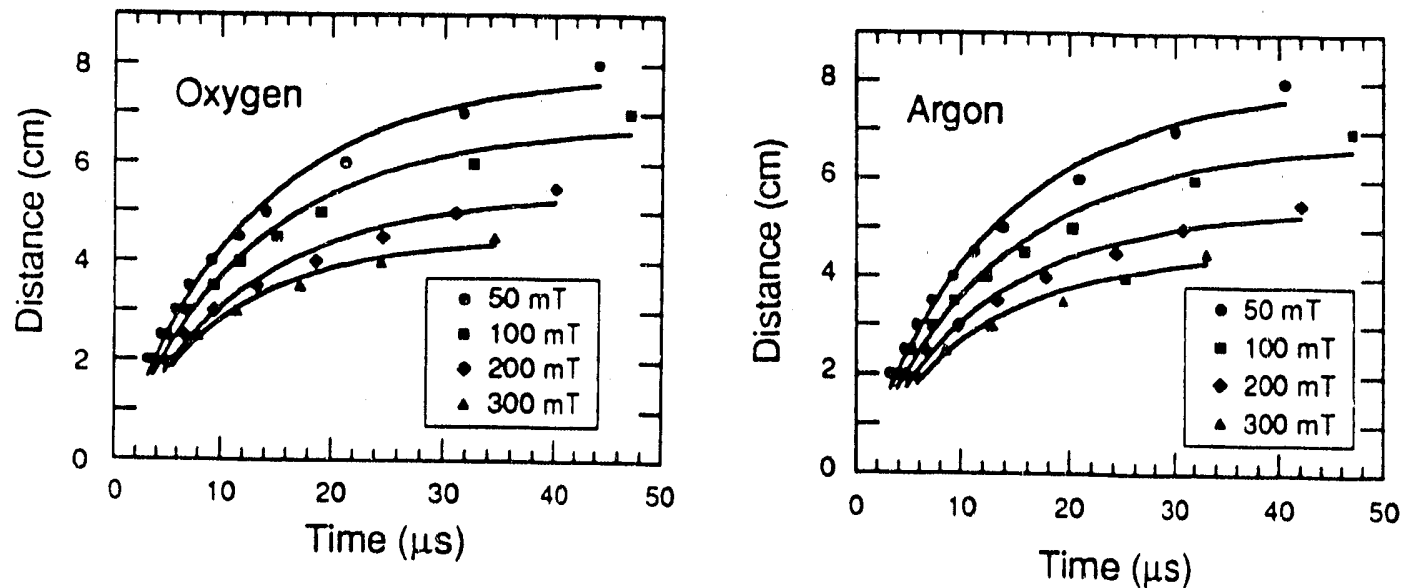

Figure 5. Position-time plots of the plasma propagation through 50,100,200, and $300 \mathrm{mT}$ oxygen and argon following $\mathrm{KrF}$ laser ablation of $\mathrm{Y}_{1} \mathrm{Ba}_{2} \mathrm{Cu}_{3} \mathrm{O}_{7}$ at $2.5 \mathrm{~J} \mathrm{~cm}^{-2}$. Fits to the curves are of the form $x=x_{f}(1-\exp (-b t))$.

The general features of the slowing of the ions show a continuous velocity decrease with distance and exponential drop with time, with the maximum range of the ions saturating at smaller distances with increasing pressure. Several models can be used to explain the observations. At high background pressures, a blast wave model has been applied to the luminous shock front caused by the expansion of laser-ablation-plasmas-. This model strictly applies when the mass of the ejected products is small compared with the mass of the background gas set in motion by the shock, a situation which does not apply here. Nevertheless, the propagation of a shock front through the background gas is described by the relation [7] $d=\xi_{0}\left(E / \rho_{0}\right)^{1 / 5} t^{2 / 5}$, where $\xi_{0}$ is a constant, $E$ is the energy deposited in the explosion, $\rho_{0}$ is the density of the background gas and $d$ and $t$ give the position and time. The $d, t$ plots of Fig. 5 can be fit by power law dependences, but do not saturate sufficiently at low pressures. The best fit values of the exponent also vary from 0.54 at $50 \mathrm{~m}$ Torr to 0.45 at $300 \mathrm{~m}$ Torr suggesting a better agreement at higher pressures.

A classical drag force model shows better agreement at low pressures and curve fits to the data are shown in Fig. 5. The ejected pulse of ablation products is regarded as an ensemble which experiences a viscous force proportional to its velocity through the background gas. The equations of motion are given by

$$
\begin{aligned}
& a=-\beta v \\
& v=v_{0} \exp (-\beta t) \\
& x=x_{f}(1-\exp (-\beta t))
\end{aligned}
$$

giving

$$
v=\beta\left(x_{f}-x\right)
$$$$
a=-\beta^{2}\left(x_{f}-x\right)
$$ 
where $\beta$ is the slowing coefficient, and $\bar{x}_{f}=v \nabla \beta$ is the stopping distance of the plume. From these equations of motion and the coefficients given in Table 1 , one can obtain the velocity and deceleration of the plasma boundary as it propagates through the background gas.

Table 1

\begin{tabular}{|c|c|c|c|c|}
\hline $\begin{array}{c}\mathrm{p} \\
(\mathrm{m} \text { Torr })\end{array}$ & $\begin{array}{c}1 / \beta(\mu \mathrm{s}) \\
\mathrm{O}_{2}\end{array}$ & $\begin{array}{c}1 / \beta(\mu \mathrm{s}) \\
\mathrm{Ar}\end{array}$ & $\begin{array}{c}\mathrm{x}_{\mathrm{f}}(\mathrm{cm}) \\
\mathrm{O}_{2}\end{array}$ & $\begin{array}{c}\mathrm{x}_{\mathrm{f}}(\mathrm{cm}) \\
\mathrm{Ar}\end{array}$ \\
\hline 50 & 13.0 & 13.2 & 7.9 & 8.0 \\
\hline 100 & 12.9 & 13.4 & 6.8 & 6.8 \\
\hline 200 & 12.1 & 12.1 & 5.4 & 5.4 \\
\hline 300 & 10.2 & 11.2 & 4.5 & 4.5 \\
\hline
\end{tabular}

\section{CONCLUSIONS}

The attenuation and slowing of the ion pulse produced by KrF laser irradiation of $\mathrm{Y}_{1} \mathrm{Ba}_{2} \mathrm{Cu}_{3} \mathrm{O}_{7}$ in background gases of argon and oxygen has been characterized using spatially resolved ion probe and optical emission measurements. The exponential decrease of ions with pressure and distance follows elastic scattering theory at low pressures. At higher pressures, inelastic effects change the velocity distribution within the beam. The ions are still attenuated exponentially, however the cross section for ion-atom or ion-molecule collisions includes several inelastic effects such as formation of $\mathrm{YO}^{*}$ and recombination.

A weak shock structure appears to be formed at the pressures used for thin film growth. The ion probe waveform represents the plasma front as it moves through the background gas. At pressures $-150 \mathrm{mTorr}$ and $\mathrm{d}>2 \mathrm{~cm}$, the leading edge of the plasma front becomes coincident with the onset of emission from the ions, neutrals and oxides in the plume and the visible emission is enhanced as the shock front inieracts with the gas ahead of it. In this pressure range, the mass of the abtated products exceeds the mass of the background gas in its path and the collisional slowing appears better represented by a viscous drag force rather than a rigid blast wave. The drag force model determines the range and velocity of the plume from distance-time plots of the plasma front.

\section{ACKNOWLEDGEMENTS}

The technical expertise and physics discussions with Doug Mashburn are gratefully acknowledged as well as research discussions with D.P. Norton and D.H. Lowndes. This research was sponsored by the Division of Materials Sciences, U.S. Department of Energy under contract DE-AC05-84OR21400 with Martin Marietta Energy Systems, Inc.

\section{REFERENCES}

1. Roger Kelly and R. W. Dreyfus, Surf. Sci. 198, 263 (1988).

2 D. B. Geohegan and D. N. Mashburn, Appl. Phys. Lett. 55, 2345 (1989).

3. D. B. Geohegan and D. N. Mashburn, in Superconductivity and Applications edited by

H. S. Kwok et al. (Plenum Press, New York, 1990), p. 153.

4. T. Venkatesan, et al., Appl. Phys. Lett. 53, 1431 (1988).

5. C. Girault, D. Damiani, J. Aubreton, and A. Catherinot, Appl. Phys. Lett. 55, 182 (1989).

6. D. B. Geohegan and D. N. Mashburn, in Laser Ablation for Materials Synthesis, Materials Research Society, San Francisco, California, 1989. (in press)

7. D. N. Mashburn and D. B. Geohegan, in Processing of Films for High-T Microelectronics, SPIE, Vol. 1187, p. 172.

8. Ya.B.Zel'dovich and Yu.P.Raizer; in Physics of Shock Waves and High-Temperature Hydrodynamic Phenomena, (Academic Press, New York 1966), vol.1, p. 94.

9. J.B. Anderson, in Molecular Beams and Low Density Gasdynamics, ed. by P.Wegener, (Marcel Dekker, New York, 1974). 

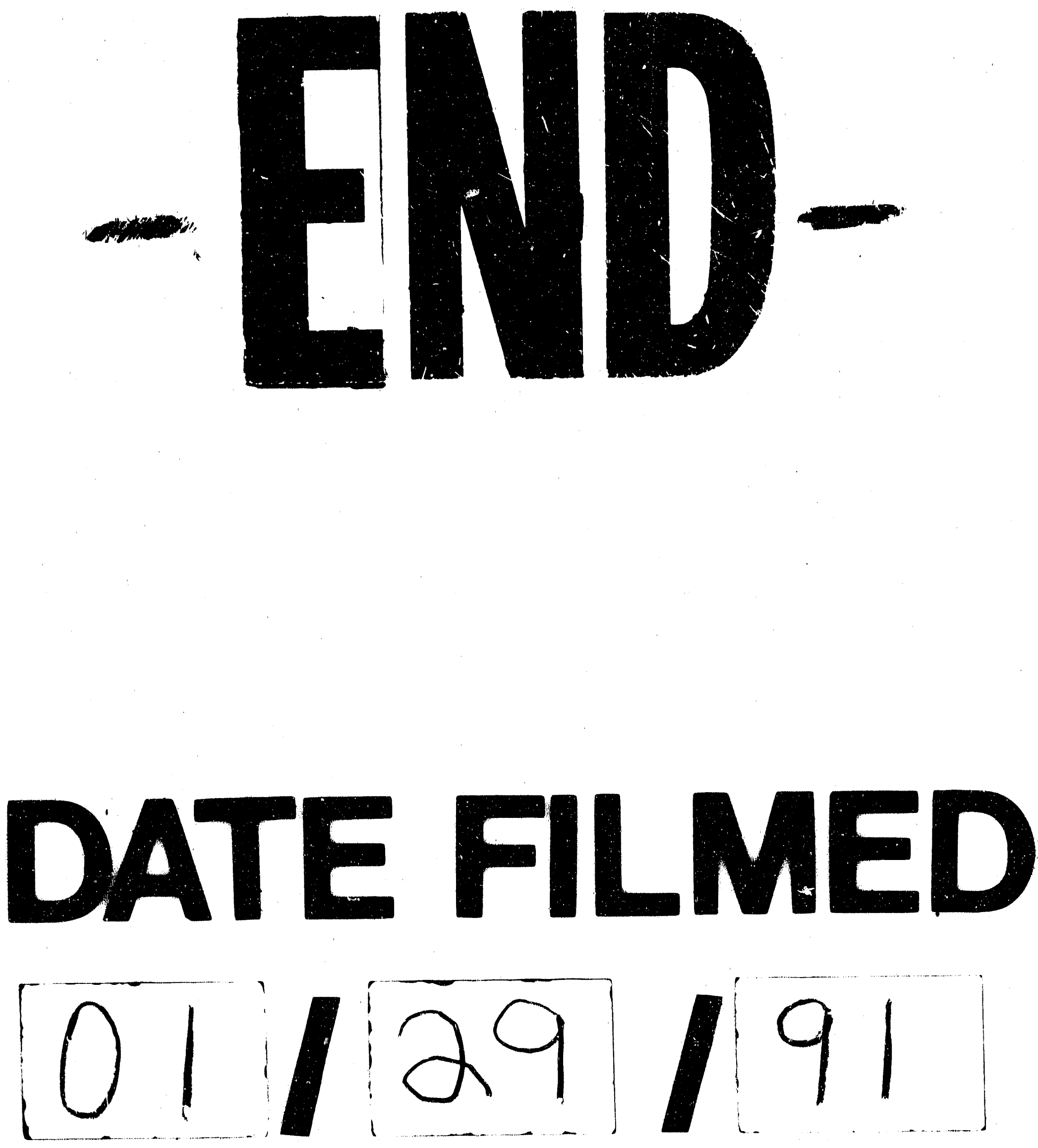


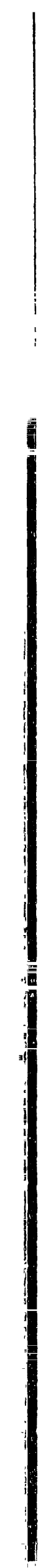

